\title{
Tumor penetrating nanomedicine targeting both an oncomiR and an oncogene in pancreatic cancer
}

\author{
Maud-Emmanuelle Gilles ${ }^{1}$, Liangliang Hao ${ }^{2,3}$, Kaelyn Brown ${ }^{1}$, Jihoon Lim¹, Sangeeta \\ N. Bhatia ${ }^{2,3,4,5,6,7}$ and Frank J. Slack ${ }^{1}$ \\ ${ }^{1}$ HMS Initiative for RNA Medicine, Department of Pathology, Cancer Center, Beth Israel Deaconess Medical Center, Harvard \\ Medical School, Boston, Massachusetts, USA \\ ${ }^{2}$ Institute for Medical Engineering and Science, Massachusetts Institute of Technology, Cambridge, Massachusetts, USA \\ ${ }^{3}$ Koch Institute for Integrative Cancer Research, Massachusetts Institute of Technology, Cambridge, Massachusetts, USA \\ ${ }^{4}$ Department of Medicine, Brigham and Women's Hospital and Harvard Medical School, Boston, Massachusetts, USA \\ ${ }^{5}$ Broad Institute of Massachusetts Institute of Technology and Harvard, Cambridge, Massachusetts, USA \\ ${ }^{6}$ Howard Hughes Medical Institute, Cambridge, Massachusetts, USA \\ ${ }^{7}$ Marble Center for Cancer Nanomedicine, Massachusetts Institute of Technology, Cambridge, Massachusetts, USA \\ Correspondence to: Frank J. Slack, email: fslack@bidmc.harvard.edu
}

Keywords: miRNAs; KRAS; RNA-based-therapy; PDAC; tumor-penetrating nanocomplexes

Received: March 28, $2019 \quad$ Accepted: July 28, $2019 \quad$ Published: September 03, 2019

Copyright: Gilles et al. This is an open-access article distributed under the terms of the Creative Commons Attribution License 3.0 (CC BY 3.0), which permits unrestricted use, distribution, and reproduction in any medium, provided the original author and source are credited.

\section{ABSTRACT}

Developing new targeted therapy for pancreatic cancer is one of the major current challenges in cancer research. KRAS mutations and miRNA dysregulation (e.g. miR-21-5p oncomiR) play key roles in Pancreatic Ductal Adenocarcinoma (PDAC), leading to rapid progression of the disease. As the KRAS mutation is a main driver of PDAC, anti-KRAS strategies remain a major therapeutic approach for PDAC treatment. Previously, utilization of either siKRAS or small chemically modified single-stranded RNA molecules that specifically disable miR-21 (anti-miR-21) were effective in slowing PDAC tumor growth in various tumor models when packaged in an innovative delivery system (TPN) required for efficient drug delivery to the PDAC tumor site. Here we have tested the utility of targeting the KRAS pathway through multiple mechanisms and via dual targeting of a PDAC oncomiR and oncogene. Initially we found that miR-217, which has been shown to directly regulate KRAS expression, is downregulated in our PDAC samples, thus we tested the benefits of anti-miR-21, miR-217 mimic or siKRAS loaded into the tumor-penetrating nanoparticles (TPN) that we had previously shown to potently target the largely impenetrable PDAC tumors, and found an enhanced antitumoral response upon dual treatments in KRAS-mutated PDAC models.

\section{INTRODUCTION}

Pancreatic cancer is currently the second leading cause of all cancer-related deaths, with a dramatically low 5 -year survival associated with a poor prognosis [1,2]. In addition to improvement of early detection, the development of potent targeted therapy methods is desperately needed. In the past, utilization of single-agent treatments like gemcitabine or combination treatments like FOLFORINOX (5-fluorouracil
(5-FU), irinotecan, oxaliplatin, and leucovorin) has allowed non-resistant patient response and increased survival for metastatic patients [1]. Combinations of multiples drugs have emerged as possible therapies and the combination of Gemcitabine with erlotinib or with nab-paclitaxel has been beneficial for patients $[3,4]$. Multiple other approaches are currently being developed for PDAC targeted therapies including the targeting of key regulators of the disease like oncogenes or small non-coding RNA (e.g. miRNAs). 
PDAC tumors are characterized by a strong desmoplastic reaction (rich stroma) that impairs easy drug delivery into the tumor site, leading to insufficient tumor targeting. Recently, various strategies have been developed to improve drug delivery to PDAC sites by utilizing tools such as nanoparticle albumin-bound paclitaxel, pseudopeptides, or polyplexes $[3,5,6]$. Additionally, various engineering approaches have led to the safe delivery of therapeutic compounds into tumor sites; among them, iRGD-guided tumor penetrating nanocomplexes (TPNs) have been specifically successful $[7,8]$. TPNs promote the safe delivery of RNA-based therapeutics including single-strand oligonucleotides $[9,10]$. We have successfully used TPNs for delivery to PDAC of an RNA-based therapeutic (antimiR-21) that targets the oncomiR (miR-21-5p) named TPN-21. TPN21 limits cell growth and increases apoptosis in various PDAC models including patient derived organoids (PDO) and patient derived xenografts (PDX), allowing its use for personalized medicine [9]. Along the same lines, due to the prevalence of KRAS mutations in PDAC, the KRAS oncogene has been targeted using an siRNA (si-KRAS) by loading it in similar particle formulations $[10,11]$. Utilization of iRGD TPNs carrying KRAS siRNA also led to the limiting of tumor growth in vivo [12]. Because miR-21 overexpression and KRAS mutation have been extensively reported to be a part of a PDAC signature with a strong clinical correlation for PDAC progression and survival $[9,10,13]$, we hypothesized that dual targeting of these two key players could improve the anti-cancer effects.

Since it seems that KRAS knockdown is unlikely to suffice as a monotherapy given the strong possibility of resistance due to compensatory mutations and altered expression profiles, it is necessary to identify and test new targets that will enhance or synergize with KRAS pathway blockades. Gene expression screens, shRNA screens and CRISPR/Cas9-powered screens are generating unprecedented lists of genetic targets that have the potential to become new RNA therapies [14].

We and other have found miR-217-5p to be downregulated in PDAC samples and its dysregulation has been found to be significantly associated with low survival in a variety of cancer types (Table 1) $[9,15]$.

miR-217 is also known to bind to the KRAS 3'UTR and impair its expression, leading to tumor suppressor activities in various cancers like acute myeloid leukemia, colorectal cancer and PDAC [16-18]. Because KRAS remains a notoriously undruggable target, here we explore various approaches such as the use of miRNA targeting $K R A S$ (miR-217 mimic) for successful combination therapy with antimir-21. As TPN approach has been successful to deliver antimiR in PDAC model, subject to validation of the anti-tumor effect of miR-217 mimic in vitro, In vivo reintroduction of the mimic will be performed through used of TPN.
In this study, we tested dual targeting of miR21 (anti-miR-21) and KRAS (siKRAS or mimic-217) packaged in TPNs for PDAC therapy. We first highlight the KRAS and miRNAs signature of our PDAC mouse model as well as the influence of $K R A S$ knock-down on miR-21 expression. We then evaluated the best approaches to target KRAS in our model by using chemically modified double-stranded RNAs that mimic endogenous miRNA miR-217 known to bind the KRAS 3'UTR and impair its expression. Then we evaluated the combination of an siRNA against KRAS and anti-mir-21 loaded into the TPN for gymnotic delivery in vitro and in an organoid model in vitro, as well as for systemic intravenous injection in vivo.

\section{RESULTS}

\section{$K R A S$ mutation and miRNA dysregulation are components of the mPDAC gene signature}

$K R A S$ mutations and alterations represent the most common abnormalities found in human PDAC samples (Figure 1A) [22, 23]. It has also been extensively reported that various miRNAs are dysregulated in PDAC in human and mouse. Among these miRNAs, some are known to target tumor suppressors or oncogenes like $K R A S$. Ten miRNAs have been validated to bind to the KRAS 3'UTR in human samples (Tarbase ref), including miR-217 (Figure 1B). Recently, our lab reported on a list of 13 miRNAs significantly deregulated in human PDX PDAC samples [9], and miR-217 was the most downregulated compared to normal samples. In this new study, we used various PDAC models including human and mouse cell lines to test the role of miR-217. In our previous study, we investigated the effect of anti-miR-21 therapy on various models including the D8-175 mouse cell line (mPDAC) derived from KrasLSL-G12D/+, p53fl/fl (KC) transgenic mice that bear the most common mutations found in PDAC patients (KRAS and TP53). We also performed an in vivo study in which NOD/SCID mice grew mPDAC allografts after subcutaneous injection of 500,000 mPDAC cells/flank. We measured miRNAs levels in tumors collected from these mice using qPCR by comparing 3 normal pancreases from mice (normal pancreas) to that of 7 mPDAC samples. As compared to our PDX human profiling, miR-217 was downregulated in this PDAC mouse model (Figure 1C) and miR-21 was upregulated (Figure 1D). These results reveal a strong miR-21 and miR-217 PDAC signature in our samples and point to a lack of $K R A S$ inhibition by its repressor: miR-217-5p.

Since we wished to test targeting of both $K R A S$ and miR-21 for PDAC therapy, we analyzed miR-21 regulation during partial knockdown of $K R A S$. To this end, mPDAC cells carrying an inducible anti-KRAS shRNA construct were cultivated under doxycycline selection for $48 \mathrm{~h}$. At the end of the treatment, we analyzed $K R A S$ expression and miR-21 expression by qPCR. KRAS expression was 
Table 1: Expression of natural KRAS targeting miRNA (miR-217-5p) is associated with poor overall survival in cancers and could be reintroduced or targeted for therapy

\begin{tabular}{lcc}
\hline Cancer Type & $\boldsymbol{P}$ Value & miR-217 expression associated low survival \\
\hline Kidney renal papillary cell carcinoma [KIRP] & $8.79 \mathrm{E}-05$ & high expression \\
Bladder Urothelial Carcinoma [BLCA] & 0.00033535 & high expression \\
Colon adenocarcinoma [COAD] & 0.00609379 & high expression \\
Kidney Chromophobe [KICH] & 0.03016767 & high expression \\
Brain Lower Grade Glioma [LGG] & $4.70 \mathrm{E}-10$ & high expression \\
Mesothelioma [MESO] & 0.00293455 & high expression \\
Stomach adenocarcinoma [STAD] & 0.02206944 & high expression \\
Ovarian serous cystadenocarcinoma [OV] & 0.06356434 & low expression \\
Testicular Germ Cell Tumors [TGCT] & 0.04835562 & low expression \\
\hline
\end{tabular}

Table 1 has been generated from the PROGmiR tool by querying miR-217-5p expression level and correlating this with overall survival ( $p$-value $£ 0.1$ ) among 33 human cancer databases. Non-significant results (p-value ${ }^{3} 0.1$ ) are not presented in the table.

decreased by $60 \%$ (Figure 1E) and miR-21 expression was also decreased by $55 \%$ (Figure $1 \mathrm{~F}$ ). In the mPDAC model (mPDAC cells carrying an inducible anti-KRAS shRNA construct grown as allografts in mice) we also observed miR-21 downregulation after mice were fed with doxycycline food to induce the shRNA KRAS (Figure 1G). These results suggest that KRAS expression influences miR-21 expression in PDAC and reinforces the idea of testing dual targeting of $K R A S$ and miR-21 as a possible therapeutic strategy.

\section{Reintroduction of miR-217 act as a tumor suppressor targeting KRAS in PDAC}

Because miR-217 was previously validated to target the KRAS 3'UTR (Figure 1B) we decided to investigate if the reintroduction of miR-217 could modulate KRAS expression in our PDAC models. To this end, we used a chemically modified double-stranded RNA that mimics endogenous miRNA miR-217 (mimic-217). hPDAC (PANC1) and mPDAC (d8-175) cells were transfected with mimic-217 for $48 \mathrm{~h}$. At the end of the treatment, expression of miR-217 was analyzed by qPCR. In both cell lines, mimic-217 induced an upregulation of miR-217 from 50 to 3000-fold (D8-175 and PANC1 respectively) (Figure 2A). Concomitantly, KRAS expression was monitored by qPCR and both cell lines showed an inhibition of KRAS expression level (50-60\%) (Figure 2B). These results show that mimic-217 represses $K R A S$ in PDAC cells.

Interestingly, we showed that $K R A S \mathrm{KD}$ promoted miR-21 downregulation in mPDAC cells (Figure $1 \mathrm{E}$ and $1 F)$. To verify if the KRAS targeting miRNA (mimic-217) promotes the same phenotype, we assessed miR-21 expression level in both cell lines after transfecting mimic-217. It appears that reintroduction of miR-217 promotes miR-21 downregulation $(\sim 40 \%)$ as observed previously after $48 \mathrm{~h}$ of shKRAS induction (Figures $2 \mathrm{C}$ and $1 \mathrm{~F}$ ). As miR-217 acts as a tumor suppressor and seems to be an interesting target for PDAC therapy we also investigated the effect of its reintroduction (alone or combined with anti-miR-21) on cell viability. After $48 \mathrm{~h}$ of transfection with either antimiR or mimic, we measured metabolic activity using an MTT assay. Analysis of the results shows a significant inhibition of viability after miR-217 reintroduction in both cell lines. On the other hand, combining anti-miR-21 and mimic-217 did not significantly influence the viability compared to each treatment used alone. These results suggest that dual targeting of these two key miRNAs (21 and 217) may not be the optimal option for PDAC therapy and suggest that direct KRAS KD using siRNA (si-KRAS) could be more appropriate than mimic-217 to impair crosstalk in miRNA/ $\mathrm{mRNA} /$ target regulations.

\section{Combining antimiR-21 and si-KRAS increases apoptosis and enhances the anti-proliferative effect in PDAC cells and organoids}

As previously demonstrated by our group, in order to optimize delivery and targeting of RNA-therapeutics to PDAC, we have encapsulated antimiRs and siRNA in tumor-penetrating nanocomplexes (TPNs) that have increase tumor targeting through sequential binding to integrins and NRP1 by tumor penetrating peptide iRGD $[9,10]$. Here we used TPNs containing anti-miR-21 (TPN21 , blue) as well as an siRNA against $K R A S$ (TPN-KRAS, red). Both TPNs were delivered by gymnotic delivery in vitro and systemic intravenous repeat injections in vivo (Figure 3A). In order to assess the anti-tumor effects of dual anti-miR-21 and si-KRAS therapy in PDAC, we treated cells using either TPN-21, TPN-KRAS, or a combination of both and we compared that to TPN-NT (scramble RNA) or double TPN-NT (combo-NT). After a 
$48 \mathrm{~h}$ treatment, we stained for apoptosis by using a specific dye that is cleaved in the nucleus under a high level of activated caspase-3. Analysis of the staining showed a significant increase of caspase- 3 activity after either TPNKRAS or TPN-21 used alone compared to TPN-NT in hPDAC and mPDAC (Figure 3B and 3C). Interestingly, an additional increase of caspase- 3 activity is observed with the combination of TPN-21 and TPN-KRAS together compared to that of individual treatments. No significant change was observed between a single and double dose of TPN-NT, indicating the low toxicity of the particles (Figure 3B and 3C).

In order to validate these results for possible human applications, PANC1 cells were plated to promote spheroid formation and relative growth rate was analyzed by performing a metabolic activity measurement using MTT. Analysis of the viability shows similar results to those previously observed in $2 \mathrm{D}$ culture including a significant increase of anti-tumor effects after dual targeting of
miR-21 and KRAS compared to TPN-KRAS alone and combo-NT (Figure 3D). Altogether these promising data suggest that dual targeting of an oncomiR (miR-21) and an oncogene (KRAS) should improve the efficiency of TPN21 and TPN-KRAS used alone for PDAC in vivo.

\section{Combining TPN-21 and TPN-KRAS shows limited benefit to the tumor growth volume but promotes tumor regression in mPDAC}

To test whether the use of dual miR-21 and KRAS targeting using RNA-based therapy can disrupt tumor maintenance, we tested our combo-therapy in vivo. We generated a PDAC mouse model by injecting 500,000 mPDAC cells/flank in NOD/SCID mice as previously described by our group [9]. As observed from our prior 3D analysis in hPDAC (Figure 3D), repeated, systemic administration of both TPN-21 and TPN-KRAS slowed tumor growth all along the treatment in $\mathrm{MPDAC}$ (not shown).
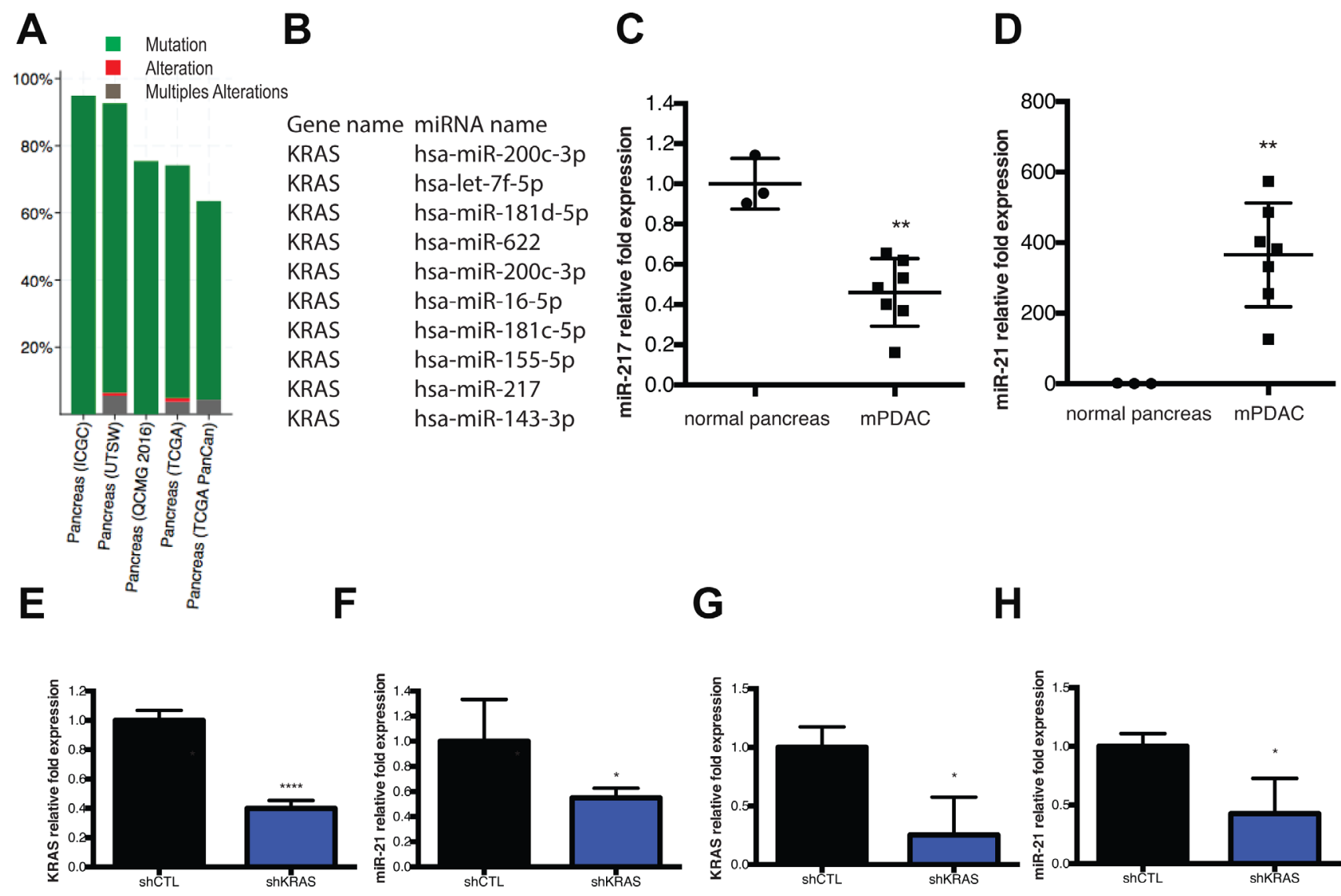

G

H
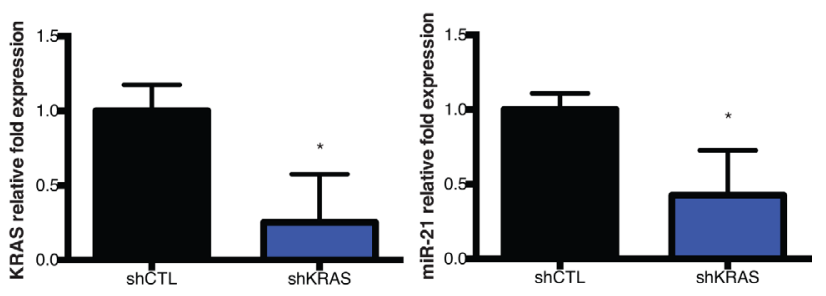

Figure 1: KRAS mutation and miRNA dysregulation are components of the mPDAC gene signature. (A) cBioPortal data presenting percentage of $K R A S$ gene mutation, alteration or multiples alteration map, from 1034 Pancreatic Adenocarcinoma samples from 5 independent studies. (B) The top 10 miRNAs that are validated to target the KRAS 3'UTR were identified in DIANA-TarBase v8. (C) qPCR analysis of miR-217-5p expression level in mPDAC tumor samples compared to normal pancreas. (D) qPCR analysis of miR-21-5p expression level in mPDAC tumors samples compared to normal pancreas. (E) qPCR analysis of KRAS expression levels in D8-175 cells treated with doxocycline for $48 \mathrm{~h}$ (shKRAS) compared to untreated D8-175 (shCTL). (F) qPCR analysis of miR-21-5p expression levels in D8-175 cells treated with doxycycline for 48h (shKRAS) compared to untreated D8-175 (shCTL). (G) qPCR analysis of miR-21-5p expression levels in mPDAC tumors from mice feed with doxycycline. ${ }^{*} P=0.01-0.05 ;{ }^{* *} P=0.001-0.01 ;{ }^{* * *} P<0.001 ;{ }^{* * * *} P<0.0001$ N.S., not significant, two-tailed $t$-test. 
In this study, tumor groups were internally heterogenous and some of TPN-NT tumors (as well as in other groups) was very small (smaller $<25 \mathrm{~mm} 3$ ) when some other are bigger $(>169 \mathrm{~mm} 3)$. Tumors sizes are not significantly different at the beginning of the study (Supplementary Figure 1).

Tumor size measurement at the end of the treatments revealed that delivery of anti-miR-21, as well as si-KRAS, significantly reduced mPDAC tumor growth by $37.3 \%$ and $37.6 \%$ respectively (Figure $4 \mathrm{~A}$ ). In addition, after dual targeting of miR-21 and KRAS (combo), the final tumor growth was reduced by $43.4 \%$, revealing an additional $6 \%$ anti-tumor effect (Figure 4A). Evaluation of the tumor growth volumes of each mouse throughout the treatment highlighted some dramatic changes in tumor growth behavior in the group receiving the combination of anti-
miR-21 and siRNA anti-KRAS. As shown (Figure 4B), only 3 tumors displayed size regression (from $17.93 \%$ to $87.59 \%$ (tumors 3 and 1 respectively)) and all 3 were in the dual targeting treatment group (Supplementary Figure 2). KRAS expression analysis of tumor samples after TPN treatments (non-targeting, TPN-siKRAS and dual treatment TPN-siKRAS+TPN-21) was performed by Western blot and we found. that the dual treatment (TPNsiKRAS + TPN-21) did not enhance KRAS KD compared to that of regular siKRAS KD (TPN-siKRAS) (Figure 4C).

This result paves the way to continue exploring how dual targeting of miR-21 and KRAS can promote tumor regression in mice and generates additional questions regarding the specific oncogene and oncomiR profile of the 3 tumors that demonstrated tumor regression.
A

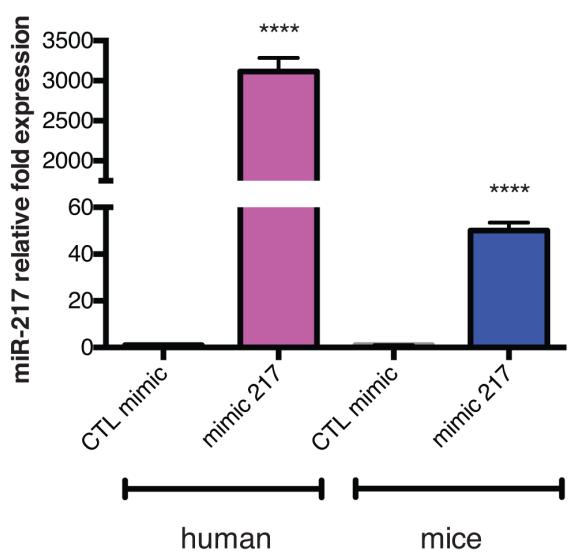

D

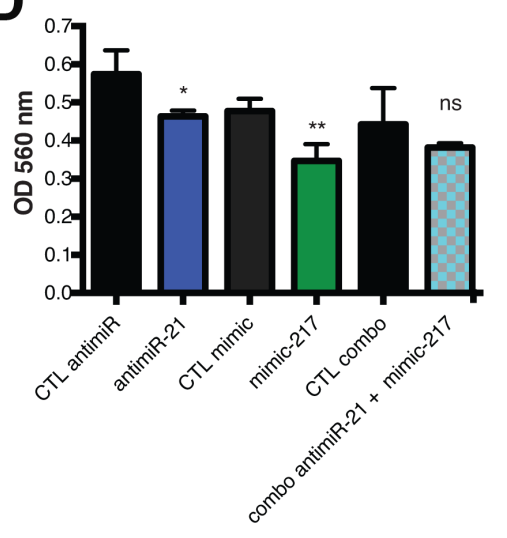

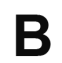

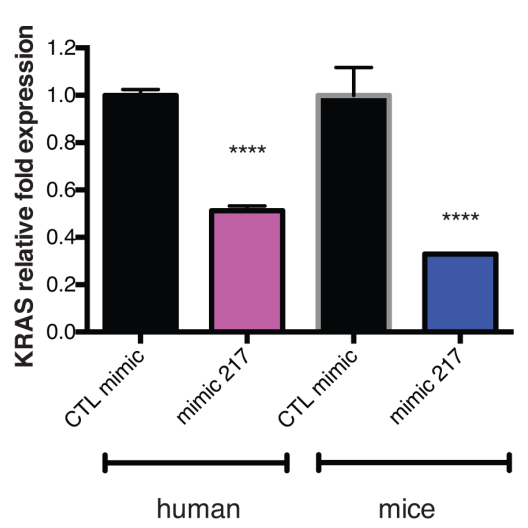

E

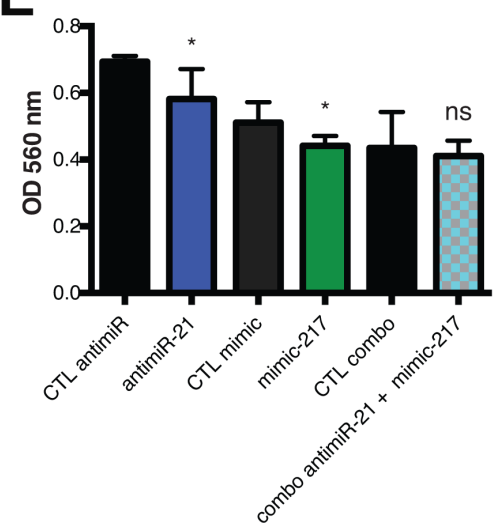

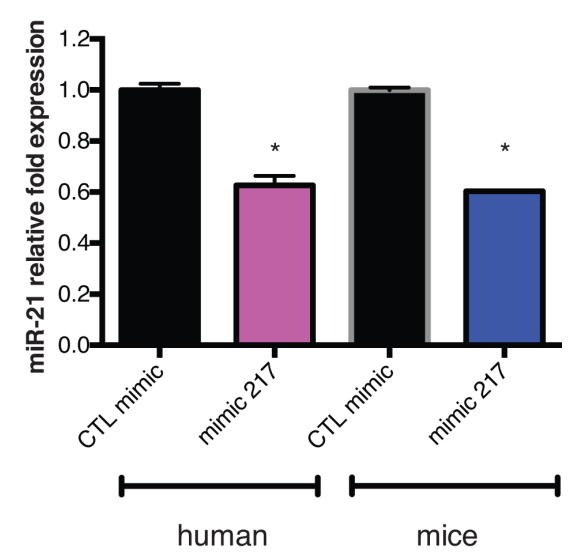

C 


\section{DISCUSSION}

In this study, we tested the dual targeting of an oncomiR (miR-21-5p) and an oncogene (KRAS) for PDAC therapy. This unique and innovative combination of compounds builds on trends in current research of successful combination approaches to PDAC therapy. To date, use of a classical chemotherapeutic agent like gemcitabine with another compound (erlotinib, nab-paclitaxel, specific pseudopeptide or small noncoding RNA strategy) has remained the first therapeutic research option for PDAC [3-6, 9]. As PDAC specimens (mouse and human samples) present with a strong $K R A S$ mutation profile associated with a strong miRNA dysregulation profile (miR-21-5p and miR-217-5p), we decided to test dual targeting of miR-21-5p and a natural KRAS targeting miRNA (miR-217-5p) (Figures 1 and 2) $[11,15]$.

Even though reintroduction of miR-217-5p using a chemically modified double-stranded RNA that mimics endogenous miRNA miR-217 (mimic-217) in mPDAC and hPDAC was successful and promoted significant KRAS knockdown and a viability defect, dual utilization of miR217-5p and anti-miR-21-5p did not yield stronger results

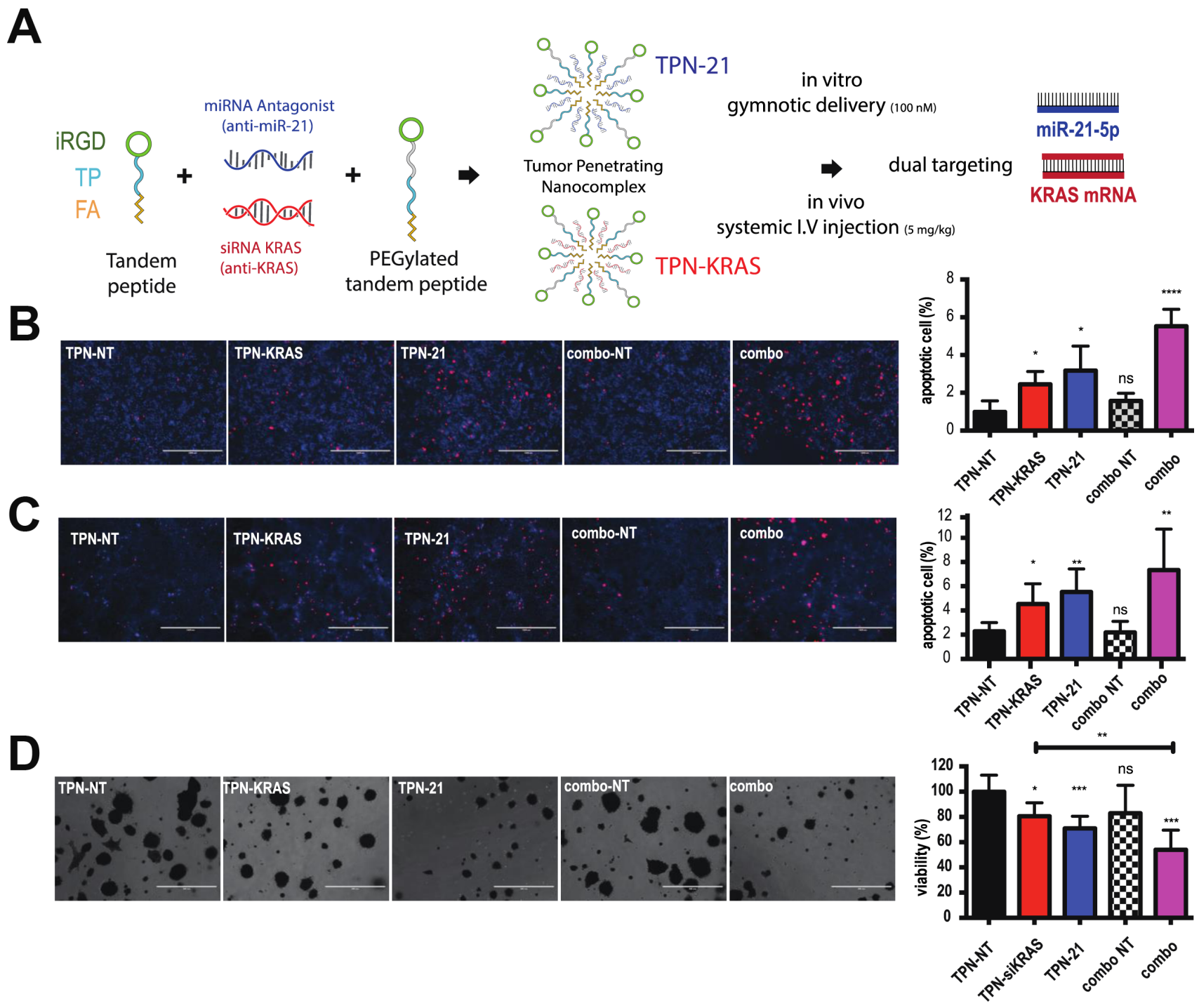

Figure 3: Combining antimiR-21 and si-KRAS increases apoptosis and enhances the anti-proliferative effect in PDAC cells and organoids. (A) Representation of tumor-penetrating nanocomplexes (TPN) loaded with anti-miR-21 (TPN-21) or loaded with siRNA-anti-KRAS (TPN-KRAS) for dual targeting of miR-21-5p and KRAS mRNA. (B and C) Representative pictures of PANC1 treated $48 \mathrm{~h}$ with TPN-21, TPN-KRAS or combo, and associated controls. Scale bars $1000 \mu \mathrm{m}$. Nuclei were stained with DAPI and NucView ${ }^{\mathrm{TM}}$ staining (red) report a significant increase in caspase-3 activity after treatments. Quantification of caspase-3 positive cells (Red) on a total cell (DAPI) is presented as apoptotic cell \%. (D) Representative images of PDO 286 metabolic activity measured from MTT assay after repeated TPN-21, TPN-KRAS or combo and associated controls treatments. Scale bars $400 \mu \mathrm{m}$. Associated quantification is presented as viability \%. Error bars, mean \pm s.d ${ }^{*} P=0.01-0.05 ;{ }^{* *} P=0.001-0.01 ;{ }^{* * *} P<0.001 ;{ }^{* * * *} P<0.0001$ N.S., not significant, two-tailed $t$-test; $n=3$ biological replicates. 
than the individual treatments (Figure 2D, 2E). These observations could be in part due to the higher toxicity of mimic control than antimiR control that decreases the viability of the double control in our assay, resulting in a minimization of the effect (Figure 2D, 2E). On the other hand, it is not surprising that indirectly targeting $K R A S$ using a miRNA promoted weaker knockdown than direct targeting with an siRNA (Figure 3B, 3C). For the rest of our combination study of oncomiR and oncogene targeting, we used an siRNA to target KRAS instead of mimic-217.

Simultaneous targeting of these two notorious PDAC drivers was achieved by using the PEGylated iRGD TPNs nanoparticle-based approach to deliver both nucleic acids (anti-miR-21 and siRNA anti-KRAS) in 2D, $3 \mathrm{D}$ and in vivo models. This compound was previously described by our groups to surmount the PDAC barrier and promote safe delivery of nucleic acid compounds [9, 10]. Our study highlights a benefit of dual targeting of these two PDAC drivers compared to single treatment and very interestingly, we even recorded 3 tumors regressing in dual-treated mice. These results indicate that dual targeting of KRAS and miR-21-5p could potentially abolish tumor growth in PDAC. Nevertheless, even though we observed a general slowing of tumor growth in the 8 mice from the combination group (combo), only 3 of them display a dramatic change in the tumor growth behavior. It's therefore unclear if the combined treatment variability is due to overall inconsistent TPN delivery to the tumor or by variability in the functional activity of the siRNAs and antimirs themselves and will be investigated in the futur. So far, we can potentially hypothesize that like humans, each mouse presents its own gene profile including specific oncogene and oncomiR dysregulation patterns and that dual targeting of miR-21-5p and $K R A S$ is not the optimal therapeutic option for all of them. In fact, personalized medicine for PDAC and cancer, in general, is currently a major challenge. As also mentioned in our previous study, there is a strong disconnect between pre-clinical work performed in cell lines and xenografts and success of clinical trials due to the heterogeneity of patient gene expression pattern including oncogene and oncomiR expression [24]. For this reason, to better
A

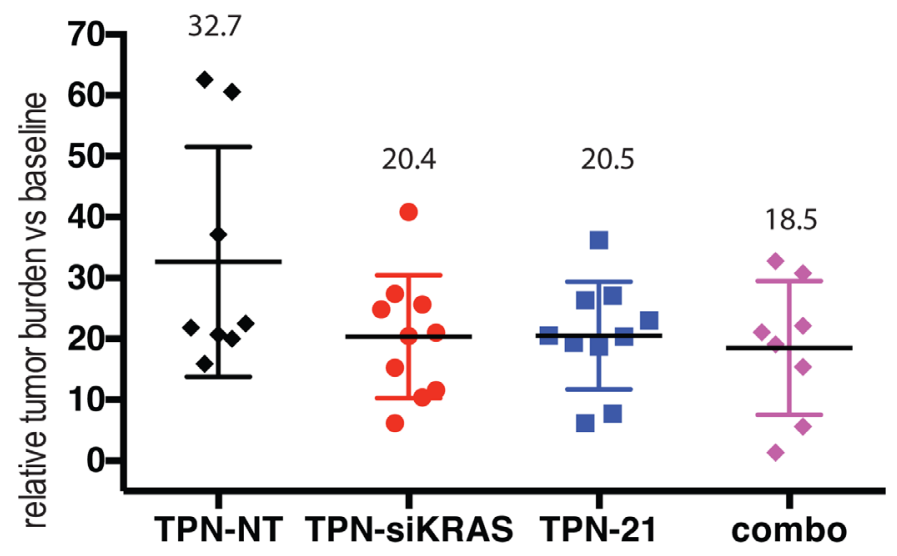

C

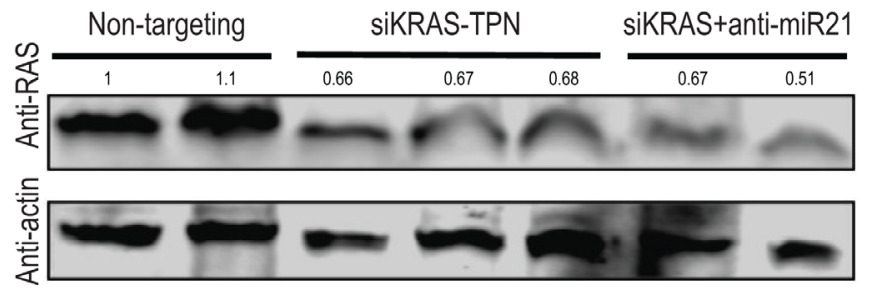

B

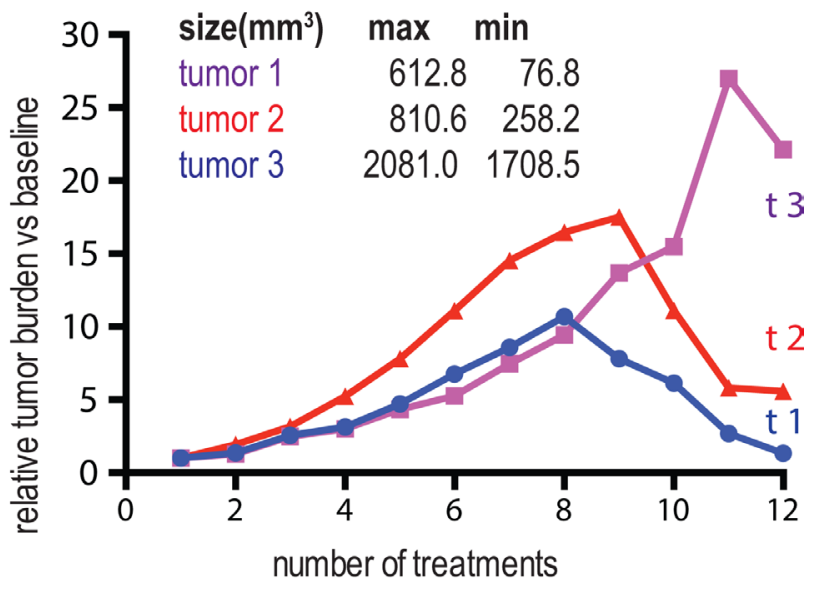

Figure 4: Combining TPN-21 and TPN-KRAS shows limited benefit to the tumor growth volume but promotes tumor regression in mPDAC. (A) Relative tumor burden in fold change after 12 I.V injections of TPN-control and combo $n=8$ or TPN-21 and TPN-KRAS $n=10(5 \mathrm{mg} / \mathrm{kg}$ ). (B) Relative tumor burden in fold change of the 3 tumors showing tumor regression (along $12 \mathrm{I} . \mathrm{V}$ injections of combo). Maximum tumor volume measured is indicated (max) as well as the tumor size at the end of the treatment (min). (C) Western blot analysis of KRAS expression, Column 1-2 (Non-targeting), columns 3-5 (TPN-siKRAS) columns 6-7 (TPN-siKRAS+TPN-21). Western blot band corresponding to KRAS expression and have been quantified against actin levels. Error bars, mean \pm s.d ${ }^{*} P=0.01-0.05$; ${ }^{* *} P=0.001-0.01 ;{ }^{* * *} P<0.001 ;{ }^{* * * *} P<0.0001$ N.S., not significant, two-tailed $t$-test; $n=3$ biological replicates. 
understand why these 3 tumor samples show regression after dual treatment we would have had to have profiled them before the therapy and then be able to understand and eventually predict tumor response. None-the-less, this specific phenotype suggests that additional work will be needed to optimize this strategy in the future.

\section{MATERIALS AND METHODS}

\section{Tumor mouse models}

For the generation of mPDAC mouse models, bilateral flank allografts were implanted on 6-week old $\mathrm{NCR} /$ nude mice each seeded with $5 \times 10^{5}$ cells. Tumor growth was monitored twice a week by observation and palpation. We defined $\sim 100 \mathrm{~mm}^{3}$ tumors as the starting point to perform treatment trials. Mice were treated 2 times a week for a total of 8 intravenous injections with either TPN21, TPN-KRAS, TPN-control (5 mg/kg oligonucleotides) or a combination (TPN-21 and TPN-KRAS). At the end of the treatment, mice were sacrificed, tumors were collected, and samples were processed appropriately. All procedures were conducted following an institutionally approved animal IACUC protocol and as described previously [9, 10].

\section{qPCR}

3D-models and organoids were separated from matrigel by washing with PBS. Cell lines and mPDAC tumors were lysed using Trizol and RNA extraction was conducted using the mirVana miRNA Isolation Kit. cDNA synthesis was performed using miScript II RT Kit (Qiagen) with $250 \mathrm{ng}$ input RNA. qRT-PCR was performed using the Roche480 Light Cycler $^{\circledR}$ miRNA system as per manufacturer's instructions, and gene expression was normalized to U6 and/or 18s. For KRAS mRNA analysis, cDNA synthesis was performed using Verso cDNA Synthesis Kit (Life technology) using 500-1000 ng input RNA. cDNA was used for SYBR Green-based real-time PCR. Gene expression was normalized to GAPDH. Hsa-miR-21-5p, mmu-miR-21-5p, Hsa-miR-217-5p, mmu-miR-217-5p, U6 and $18 \mathrm{~s}$ miScript Primers were obtained from Qiagen.

The KRAS primers used for mouse and human are F: AGAGGACTCCTACAGGAAACAAGTAGTAATTGAT R:AGCCCTCCCCAGTTCTCATGT

\section{Cell lines}

Human PDAC cells PANC-1, were obtained from the American Type Culture Collection (ATCC), and cultured at $37^{\circ} \mathrm{C}$ with $5 \% \mathrm{CO}_{2}$ in DMEM, supplemented with $10 \%$ FBS and 1\% penicillin/streptomycin. Mouse PDAC cell line D8-175 is derived from Pdx1-Cre; $\mathrm{Kras}^{+/ L S L-G 12 D}$; Trp53 ${ }^{\text {flfl }}$ (KPC) mice, and was cultured in DMEM supplemented with $10 \%$ FBS and 1\% penicillin/ streptomycin. For in vivo tumor development tracking, D8175 cells were $l u c+$ (luciferized) and carried a doxycycline inducible shRNA against $K R A S$. Cell lines were tested for mycoplasma using the Lonza MycoAlert ${ }^{\mathrm{TM}}$ Detection Kit.

\section{AntimiR, mimic and siRNA transfection}

For RNA-based functional experiments, mirVana ${ }^{\circledR}$ miRNA inhibitor for hsa-miR-21-5p and mmu-miR-21$5 \mathrm{p}$ and $\operatorname{mir} \operatorname{Vana}^{\circledR}$ miRNA mimic for hsa-miR-217-5p and mmu-miR-217-5p as well as controls mirVana ${ }^{\mathrm{TM}}$ miRNA Inhibitor and mirVana ${ }^{\mathrm{TM}}$ miRNA Mimic, Negative Control \#1 (Life Technologies) were used at $50 \mathrm{nM}$ and transfected into cells using RNAiMAX (invitrogen), as per manufacturer's protocol. KRAS siRNAs were synthesized by Dharmacon (GE Healthcare) with ONTARGETplus specificity enhancement. The following sequences were used (given as the sense strand without overhangs): siKras.476 against murine and human KRAS: 5'-ACCAUUAUAGAGAACAAAUUA-3', siNC nontargeted control: 5'-UUCUCCGAACGUGUCACG UUU-3'.

\section{Organoids}

Tumor organoids were generated as described by Huang et al 2015 [19]. Fresh PDX tumor fragments were minced with a No.22 blade into 1-2 $\mathrm{mm}$ small pieces then digested with $1 \mathrm{mg} / \mathrm{ml}$ collagenase/dispase for 30 minutes, followed by Accutase digestion for 40 minutes. The slurry was re-suspended in DMEM then filtered through a tissue strainer and centrifuged at $1500 \mathrm{rpm}$ for 5 minutes. After aspirating the supernatant, cell pellets were re-suspended in organoid growth medium supplemented with 5\% Matrigel and $10 \mathrm{uM} \mathrm{Y27632.} \mathrm{Organoids} \mathrm{were} \mathrm{grown} \mathrm{under} \mathrm{standard}$ culture conditions $\left(5 \% \mathrm{CO} 2\right.$, at $\left.37^{\circ} \mathrm{C}\right)$. Organoids were treated with TPN-21, TPN-KRAS, TPN-control or combo $(100 \mathrm{nM})$ following a QOD treatment plan.

\section{Viability and apoptotic assay}

Viability assay on cells and organoids was performed by using MTT reagents from SIGMA (M2128) according to the manufacturer's recommendation. The apoptosis assay was performed by using NucView ${ }^{\mathrm{TM}} 530$ Caspase-3 Substrate (Biotium). 3D-models were briefly incubated with NucView reagent (1/500) and Hoescht (1/1000) for 30-60 minutes and the red signal corresponding to caspase- 3 positive structures were counted and normalized to the total number of structures. Viability and apoptosis of 3D-models and organoids after treatment was conducted on $>3$ independent wells per condition in each experiment for $>3$ independent experiments.

\section{Tumor penetrating nanocomplex and binding assay}

Tandem peptide (pTP-iRGD: $\mathrm{CH} 3(\mathrm{CH}) 15-$ GWTLNSAGYLLGKINLKALAALAKKIL-GGK 
(TAMRA)GGCRGDKGPDC, Cys-Cys bridge) was synthesized by CPC Scientific. PEGylation method of the tandem peptide was previously described [20]. All siRNAs were synthesized by Dharmacon (GE Healthcare) with ON-TARGETplus specificity enhancement. The sequences used were as follows (sense strand, without overhangs): siKras: 5'-GGAAACCUUCUUUUUUCUAAG-3', siNC non-targeted control: 5'-UUCUCCGAACGUGUCA CGUUU-3'. mirVana ${ }^{\mathrm{TM}}$ miRNA inhibitor against hsamiR-21-5p and non-targeting controls were obtained from Thermo Scientific. To assembly the TPNs in solution, oligonucleotides, PEGylated pTP-iRGD, and pTP-iRGD were resuspended in nuclease-free water and mixed with the oligonucleotides, PEG-containing component, and peptide in 1:2.5:15 molar ratios. This was achieved by first thoroughly mixing the oligonucleotides with the PEGylated pTP-iRGD and subsequently mixing in the peptide to create a concentrated solution of TPNs that were adjusted to the desired dilution and buffer composition with appropriate diluent. Binding of iRGD-TAMRA on cells was performed at $4^{\circ} \mathrm{C}$ on ice.

\section{Western blotting}

Subcutaneous tumors driven by D8-175 cell line were isolated, cryogrinded and lysed in $1 x$ RIPA buffer with protease and phosphatase inhibitor (Thermo Scientific) for 30 minutes on ice. The wholecell lysates were then clarified by centrifugation for 25 minutes at $15,000 \mathrm{rpm}$ at $4^{\circ} \mathrm{C}$. Protein concentrations were determined using the BCA Protein Assay Kit (Pierce). Equal amounts $(30 \mu \mathrm{g})$ of protein samples were fractionated by a Novex ${ }^{\circledR} 4-20 \%$ Tris-Glycine gel (Thermo Scientific), transferred to nitrocellulose membranes (Thermo Scientific), and blocked in Odyssey blocking buffer (LI-COR Biosciences). KRAS was probed by primary rabbit antibody (1:1000) (Cell Signaling, \#3965), and actin was stained using primary rabbit antibody (1:1000) (Abcam, ab8227). The desired bands were detected by labeling with anti-rabbit $(1: 10,000)$ IgGIRDye 680 secondary antibodies and visualized using the Odyssey Infrared Imaging System (LI-COR Biosciences).

\section{Statistical analysis}

Statistical analysis was achieved by using a twotailed unpaired $t$ test on GraphPad Prism software. 2-way ANOVA were completed for miRNA profiling assays. All graphs show means \pm S.D ${ }^{*} P=0.01-0.05 ;{ }^{* *} P=0.001-$ $0.01 ;{ }^{* * *} P<0.001 ;{ }^{* * * *} P<0.0001$ N.S., not significant. For qRT-PCR data, means and s.d. were calculated at the $\Delta \Delta \mathrm{Ct}$ level before being converted to fold changes as presented in the graphs [21].

\section{CONFLICTS OF INTEREST}

The authors declare no conflicts of interest.

\section{FUNDING}

This work was supported by a grant to FJS and SB by the Harvard Medical School Initiative for RNA Medicine and to FJS from the BIDMC CAO Pilot Award.

\section{REFERENCES}

1. Smigiel JM, Parameswaran N, Jackson MW. Targeting Pancreatic Cancer Cell Plasticity: The Latest in Therapeutics. Cancers (Basel). 2018; 10:E14. https://doi. org/10.3390/cancers 10010014. [PubMed]

2. Hidalgo M, Cascinu S, Kleeff J, Labianca R, Löhr JM, Neoptolemos J, Real FX, Van Laethem JL, Heinemann V. Addressing the challenges of pancreatic cancer: future directions for improving outcomes. Pancreatology. 2015; 15:8-18. https://doi.org/10.1016/j.pan.2014.10.001. [PubMed]

3. Moore MJ, Goldstein D, Hamm J, Figer A, Hecht JR, Gallinger S, Au HJ, Murawa P, Walde D, Wolff RA, Campos D, Lim R, Ding K, et al, and National Cancer Institute of Canada Clinical Trials Group. Erlotinib plus gemcitabine compared with gemcitabine alone in patients with advanced pancreatic cancer: a phase III trial of the National Cancer Institute of Canada Clinical Trials Group. J Clin Oncol. 2007; 25:1960-66. https://doi.org/10.1200/ JCO.2006.07.9525. [PubMed]

4. Von Hoff DD, Ervin T, Arena FP, Chiorean EG, Infante J, Moore M, Seay T, Tjulandin SA, Ma WW, Saleh MN, Harris M, Reni M, Dowden S, et al. Increased survival in pancreatic cancer with nab-paclitaxel plus gemcitabine. $\mathrm{N}$ Engl J Med. 2013; 369:1691-703. https://doi.org/10.1056/ NEJMoa1304369. [PubMed]

5. Gilles ME, Maione F, Cossutta M, Carpentier G, Caruana L, Di Maria S, Houppe C, Destouches D, Shchors K, Prochasson C, Mongelard F, Lamba S, Bardelli A, et al. Nucleolin targeting impairs the progression of pancreatic cancer and promotes the normalization of tumor vasculature. Cancer Res. 2016; 76:7181-93. https://doi. org/10.1158/0008-5472.CAN-16-0300. [PubMed]

6. Diamantopoulou Z, Gilles ME, Sader M, Cossutta M, Vallée B, Houppe C, Habert D, Brissault B, Leroy E, Maione F, Giraudo E, Destouches D, Penelle J, et al. Multivalent cationic pseudopeptide polyplexes as a tool for cancer therapy. Oncotarget. 2017; 8:90108-22. https://doi. org/10.18632/oncotarget.21441. [PubMed]

7. Sugahara KN, Teesalu T, Karmali PP, Kotamraju VR, Agemy L, Girard OM, Hanahan D, Mattrey RF, Ruoslahti E. Tissue-penetrating delivery of compounds and nanoparticles into tumors. Cancer Cell. 2009; 16:510-20. https://doi.org/10.1016/j.ccr.2009.10.013. [PubMed]

8. Teesalu T, Sugahara KN, Ruoslahti E. Tumor-penetrating peptides. Front Oncol. 2013; 3:216. https://doi.org/10.3389/ fonc.2013.00216. [PubMed]

9. Gilles ME, Hao L, Huang L, Rupaimoole R, LopezCasas PP, Pulver E, Jeong JC, Muthuswamy SK, Hidalgo 
M, Bhatia SN, Slack FJ. Personalized RNA-medicine for pancreatic cancer. Clin Cancer Res. 2018; 24:1734 47. $\quad$ https://doi.org/10.1158/1078-0432.CCR-17-2733. [PubMed]

10. Lo JH, Hao L, Muzumdar MD, Raghavan S, Kwon EJ, Pulver EM, Hsu F, Aguirre AJ, Wolpin BM, Fuchs CS, Hahn WC, Jacks T, Bhatia SN. iRGD-guided tumorpenetrating nanocomplexes for therapeutic siRNA delivery to pancreatic cancer. Mol Cancer Ther. 2018; 17:237788. https://doi.org/10.1158/1535-7163.MCT-17-1090. [PubMed]

11. Mueller S, Engleitner T, Maresch R, Zukowska M, Lange S, Kaltenbacher T, Konukiewitz B, Öllinger R, Zwiebel M, Strong A, Yen HY, Banerjee R, Louzada S, et al. Evolutionary routes and KRAS dosage define pancreatic cancer phenotypes. Nature. 2018; 554:62-68. https://doi. org/10.1038/nature25459. [PubMed]

12. Ali S, Saleh H, Sethi S, Sarkar FH, Philip PA. MicroRNA profiling of diagnostic needle aspirates from patients with pancreatic cancer. Br J Cancer. 2012; 107:1354-60. https:// doi.org/10.1038/bjc.2012.383. [PubMed]

13. di Magliano MP, Logsdon CD. Roles for KRAS in pancreatic tumor development and progression. Gastroenterology. 2013; 144:1220-29. https://doi. org/10.1053/j.gastro.2013.01.071. [PubMed]

14. Tsherniak A, Vazquez F, Montgomery PG, Weir BA, Kryukov G, Cowley GS, Gill S, Harrington WF, Pantel S, Krill-Burger JM, Meyers RM, Ali L, Goodale A, et al. Defining a Cancer Dependency Map. Cell. 2017; 170:564576. https://doi.org/10.1016/j.cell.2017.06.010. [PubMed]

15. Müller S, Raulefs S, Bruns P, Afonso-Grunz F, Plötner A, Thermann R, Jäger C, Schlitter AM, Kong B, Regel I, Roth WK, Rotter B, Hoffmeier K, et al. Next-generation sequencing reveals novel differentially regulated mRNAs, lncRNAs, miRNAs, sdRNAs and a piRNA in pancreatic cancer. Mol Cancer. 2015; 14:94. https://doi.org/10.1186/ s12943-015-0358-5. [PubMed]

16. Zhao WG, Yu SN, Lu ZH, Ma YH, Gu YM, Chen J. The miR-217 microRNA functions as a potential tumor suppressor in pancreatic ductal adenocarcinoma by targeting KRAS. Carcinogenesis. 2010; 31:1726-33. https:// doi.org/10.1093/carcin/bgq160. [PubMed]

17. Zhang N, Lu C, Chen L. miR-217 regulates tumor growth and apoptosis by targeting the MAPK signaling pathway in colorectal cancer. Oncol Lett. 2016; 12:4589-97. https://doi. org/10.3892/ol.2016.5249. [PubMed]

18. Xiao Y, Deng T, Su C, Shang Z. MicroRNA 217 inhibits cell proliferation and enhances chemosensitivity to doxorubicin in acute myeloid leukemia by targeting KRAS. Oncol Lett. 2017; 13:4986-94. https://doi.org/10.3892/ol.2017.6076. [PubMed]

19. Huang L, Holtzinger A, Jagan I, BeGora M, Lohse I, Ngai N, Nostro C, Wang R, Muthuswamy LB, Crawford HC, Arrowsmith C, Kalloger SE, Renouf DJ, et al. Ductal pancreatic cancer modeling and drug screening using human pluripotent stem cell- and patient-derived tumor organoids. Nat Med. 2015; 21:1364-71. https://doi.org/10.1038/ $\underline{\text { nm.3973. }}$ [PubMed]

20. Lo JH, Kwon EJ, Zhang AQ, Singhal P, Bhatia SN. Comparison of Modular PEG Incorporation Strategies for Stabilization of Peptide-siRNA Nanocomplexes. Bioconjug Chem. 2016; 27:2323-31. https://doi.org/10.1021/acs. bioconjchem.6b00304. [PubMed]

21. Livak KJ, Schmittgen TD. Analysis of relative gene expression data using real-time quantitative PCR and the 2(-Delta Delta C(T)) Method. Methods. 2001; 25:402-08. https://doi.org/10.1006/meth.2001.1262. [PubMed]

22. Gao J, Aksoy BA, Dogrusoz U, Dresdner G, Gross B, Sumer SO, Sun Y, Jacobsen A, Sinha R, Larsson E, Cerami E, Sander C, Schultz N. Integrative analysis of complex cancer genomics and clinical profiles using the cBioPortal. Sci Signal. 2013; 6:pl1. https://doi.org/10.1126/ scisignal.2004088. [PubMed]

23. Cerami E, Gao J, Dogrusoz U, Gross BE, Sumer SO, Aksoy BA, Jacobsen A, Byrne CJ, Heuer ML, Larsson E, Antipin Y, Reva B, Goldberg AP, et al. The cBio cancer genomics portal: an open platform for exploring multidimensional cancer genomics data. Cancer Discov. 2012; 2:401-04. https://doi.org/10.1158/2159-8290.CD-12-0095. [PubMed]

24. Byrne AT, Alférez DG, Amant F, Annibali D, Arribas J, Biankin AV, Bruna A, Budinská E, Caldas C, Chang DK, Clarke RB, Clevers H, Coukos G, et al. Interrogating open issues in cancer precision medicine with patient-derived xenografts. Nat Rev Cancer. 2017; 17:254-68. https://doi. org/10.1038/nrc.2016.140. [PubMed] 